\title{
Envolvimento hepático em pacientes com dengue hemorrágico: manifestação rara?
}

\author{
Liver involvement in patients with dengue hemorrhagic \\ fever: a rare phenomenon?
}

\author{
Patrícia Moreira Uehara ${ }^{1}$, Rivaldo Venâncio da Cunha ${ }^{1}$, Gracy Regina Oliveira L. Pereira ${ }^{1}$ \\ e Priscilla Alexandrino de Oliveira ${ }^{1}$
}

\begin{abstract}
RESUMO
As manifestações hepáticas são descritas como não usuais no dengue e podem evoluir com quadros graves e potencialmente letais. Avaliamos as alterações hepáticas em 41 pacientes com dengue hemorrágico com confirmação laboratorial (ELISA IgM positivo) em Campo Grande, Mato Grosso do Sul, Brasil e observamos 61\% (25/41) de alteração na alanina aminotransferase e 80,5\% (33/41) na aspartato aminotransferase, sendo que não houve diferenças estatisticamente significativas quando comparamos as várias formas clínicas. A variação nos valores de ALT foi de 14-547U/1, nos valores da AST foi de 11-298U/1. Náuseas e/ou vômitos foram referidos por 90\% (37/41) dos pacientes, 46,3\% (19/41) referiram dor abdominal e 10\% (3/29) apresentavam hepatomegalia a exame físico. A idade variou de 18 a 88 anos, 23 (56\%) eram mulheres e 18 (44\%) homens.
\end{abstract}

Palavras-chaves: Dengue. Dengue hemorrágico. Alterações hepáticas. Mato Grosso do Sul State. Brasil.

\begin{abstract}
Hepatic manifestations are described as unusual complications of dengue and may lead to severe and potentially lethal conditions. Liver abnormalities in 41 patients diagnosed with dengue hemorrhagic fever in Campo Grande, Mato Grosso do Sul, Brazil, between January 1 and March 31, 2002, were evaluated. All were serologically positive for dengue in laboratory tests (IgM ELISA). ALT alterations were observed in 61\% (25/41) and AST alterations in 80.5\% (33/41), but there were no statistically significant differences between the various clinical forms. The range in ALT levels was 14-547U/1 and in AST levels was 11-298U/. Nausea and/or vomiting were reported by 90\% (37/41) of the patients; $46.3 \%$ (19/41) had abdominal pain and 10\% (3/29) presented hepatomegaly at clinical examination. The patients' ages ranged from 18 to 88 years; 23 (56\%) were female and 18 (44\%) were male.
\end{abstract}

Key-words: Dengue. Dengue hemorrhagic fever. Hepatic alterations. Mato Grosso do Sul State. Brazil.

0 dengue é uma arbovirose causada por um dos quatro sorotipos do vírus dengue (DENV-1, DENV-2, DENV-3, DENV-4) $)^{1017}$, os quais pertencem ao gênero Flavivirus da família Flaviviridae ${ }^{20}$. A doença é transmitida pela picada da fêmea infectada do mosquito Aedes aegypti (Linnaeus, 1762), espécie altamente doméstica e adaptada às condições urbanas de vida propiciadas pela sociedade moderna ${ }^{5}$.

No Brasil, a doença está presente em todos os estados, com exceção do Rio Grande do Sul e Santa Catarina, com circulação de 3 sorotipos do dengue em grande parte do território nacional. A ampla circulação viral é um fator de risco para o surgimento de formas mais graves do dengue ${ }^{89}$.
No Mato Grosso do Sul, o DENV-1 foi isolado em $1987^{3} \mathrm{e}$ os primeiros casos autóctones de dengue clássico foram notificados à Fundação Nacional de Saúde (FUNASA) em janeiro de 1990, quando o estado registrou 9.757 casos de dengue. 0 primeiro caso de dengue hemorrágico (DH) ocorreu em 1995 na cidade de Campo Grande, capital do estado, ano em que ocorreu uma grande epidemia causada pelo DENV-1, quando foram confirmados laboratorialmente 2.318 casos $^{3}$. À época deste estudo havia a circulação dos sorotipos 1, 2 no estado.

0 dengue pode, também, apresentar-se em formas clínicas atípicas $^{414}$, dentre estas, têm destaque as alterações hepáticas.

1. Serviço de infectologia do Hospital Universitário da Universidade Federal de Mato Grosso do Sul, Campo Grande, MS.

Endereço para correspondência: Dr. Rivaldo Venâncio da Cunha. Rua dos Prismas 31, Bairro TV Morena, $79050-090$ Campo Grande, MS

e-mail: rivaldo_venancio@uol.com.br

Recebido para publicação em 3/12/2006

Aceito em 4/12/2006 
Com o objetivo de estudar esta forma clínica de apresentação do dengue, ainda descrita como rara ou não usual, desenvolvemos este trabalho.

\section{CASUÍSTICA E MÉTODOS}

0 estudo consiste numa série de 41 casos de pacientes com dengue hemorrágico internados em três hospitais públicos de Campo Grande, Mato Grosso do Sul, durante o período de $1^{\circ}$ de janeiro a 31 de março de 2002.

Critérios de inclusão. Clínicos: para o diagnóstico clínico de DH foram utilizados os critérios propostos pela Organização Mundial de Saúde ${ }^{21}$. Laboratoriais: sorologia positiva para o DENV pelo teste imunoenzimático de captura de IgM (Mac-ELISA) e realização durante a internação de algum dos seguintes exames para avaliação da função hepática: ALT, AST, GGT, FA, PT (proteínas totais) e ALB. (albumina), para as enzimas foram considerados alterados os valores acima do normal e para as proteínas totais e albumina foram considerados alterados os valores abaixo do intervalo de normalidade. A data da coleta dos exames da função hepática variou do $2^{\circ}$ ao $11^{\circ}$ dia do início dos sintomas. 0 sangue coletado para a realização da sorologia para 0 dengue foi posteriormente recuperado e em 33 amostras foi realizada a sorologia para a hepatite $\mathrm{C}$ (anti-HCV) e em 32 amostras a sorologia para a hepatite B (HBSAg), em 8 pacientes as alíquotas não continham material suficiente para realização de sorologia para hepatite $\mathrm{C}$ e em 9 pacientes as amostras foram insuficientes para a realização de HBSAg.

Análise dos dados. As informações dos questionários foram processadas no programa Epi-Info 6.04 e análise estatística dos resultados foi realizada pelo teste do $\chi^{2}$ (quiquadrado) com intervalo de confiança de $95 \%$.

Após a leitura e aceite do termo de consentimento livre e esclarecido os pacientes respondiam a um questionário conduzido pelo entrevistador e tinham os dados de exame físico de entrada e exames laboratoriais coletados de seus prontuários. 0 projeto de pesquisa foi aprovado pelo Comitê de Ética em Pesquisas Envolvendo Seres Humanos da UFMS.

\section{RESULTADOS}

No total, foram estudados 41 casos e as formas clínicas assim se distribuíram: 3 casos de DH grau I, 25 casos de DH grau II, 12 casos de DH grau III e 1 caso de DH grau IV. A idade dos pacientes variou de 18 a 88 anos, com média de 41,8 anos. Quanto ao sexo, 23 (56\%) eram do sexo feminino e 18 (44\%) do sexo masculino, observamos 61\% (25/41) de alteração na ALT, 80,5\% (33/41) de alteração na AST, 40\% (8/20) na GGT, $18 \%$ (4/22) na fosfatase alcalina, 30,3\% (10/ 33) nas proteínas totais e 48,4\% (16/33) de alteração na dosagem de albumina sérica. A variação dos valores de ALT foi de 14-547U/1, com média de 89,7U/1, a variação dos valores da AST foi de 11-298U/1, com média de 104,5U/1, a mediana para ambas as enzimas foi de 96U/1.
As manifestações clínicas investigadas, que poderiam estar relacionadas a alterações da função hepática, foram dor abdominal, náuseas e vômitos e hepatomegalia; observou-se que 90\% (37/41) dos pacientes queixavam-se de náuseas e vômitos, destes $81 \%(30 / 37)$ apresentavam alteração na AST e $62,1 \%$ (23/37) tinham alteração na ALT, 46,3\% (19/41) referiam dor abdominal, sendo que destes 94,7\% (18/19) apresentavam AST alterada e 68,4\% (13/19) tinham ALT alterada. Com relação a hepatomegalia 10,3\% (3/29) a apresentavam ao exame físico, destes $66,7 \%$ (2/3) tinham alteração de AST e ALT, como está informação foi considerada na admissão do caso, nem todos os pacientes tinham este dado anotado na ficha de primeiro atendimento (Tabela 1).

A análise da correlação entre a forma clínica e as alterações das enzimas hepáticas mostrou que dos 3 pacientes com DH I 66,7\% (2/3) apresentavam alteração na AST e ALT, para o DH II encontramos 88\% (22/25) de alteração para AST e 64\% (16/25) para ALT, no DH III 66,7\% (8/12) de alteração na AST e 50\% (6/12) para a ALT e o único paciente com DH IV tinha alteração em ambas as enzimas (AST 110U/1 e ALT 71U/1). Não encontramos diferenças estatisticamente significativas entre a forma clínica e as alterações hepáticas (Tabela 2).

Tanto as mulheres como os homens apresentaram dano hepático expresso e medido pela porcentagem de casos com alterações nas enzimas, encontramos 52,1\% (12/23) para a

\begin{tabular}{|c|c|c|c|c|c|c|c|c|}
\hline \multirow{4}{*}{$\begin{array}{l}\text { Sinais } \\
\mathrm{e} \\
\text { Sintomas }\end{array}$} & \multicolumn{4}{|c|}{ ALT/TGP } & \multicolumn{4}{|c|}{ AST/TGO } \\
\hline & \multirow{2}{*}{\multicolumn{2}{|c|}{$\begin{array}{c}\text { normal } \\
\mathrm{n}=16\end{array}$}} & \multirow{2}{*}{\multicolumn{2}{|c|}{$\begin{array}{c}\text { alterada } \\
\mathrm{n}=25\end{array}$}} & \multirow{2}{*}{\multicolumn{2}{|c|}{$\begin{array}{c}\text { normal } \\
\mathrm{n}=8\end{array}$}} & \multirow{2}{*}{\multicolumn{2}{|c|}{$\begin{array}{c}\text { alterada } \\
\mathrm{n}=33\end{array}$}} \\
\hline & & & & & & & & \\
\hline & $\mathrm{n}^{0}$ & $\%$ & $\mathrm{n}^{0}$ & $\%$ & $\mathrm{n}^{0}$ & $\%$ & $\mathrm{n}^{0}$ & $\%$ \\
\hline \multicolumn{9}{|c|}{ Hepatomegalia } \\
\hline $\operatorname{sim}$ & 1 & 6,3 & 2 & 8,0 & 1 & 12,5 & 3 & 7,7 \\
\hline não & 12 & 75,0 & 14 & 56,0 & 6 & 75,0 & 24 & 61,5 \\
\hline ignorado & 3 & 18,7 & 9 & 36,0 & 1 & 12,5 & 12 & 30,8 \\
\hline \multicolumn{9}{|c|}{ Náuseas/vômitos } \\
\hline sim & 14 & 87,5 & 23 & 92,0 & 7 & 87,5 & 30 & 91,0 \\
\hline não & 2 & 12,5 & 2 & 8,0 & 1 & 12,5 & 3 & 9,0 \\
\hline \multicolumn{9}{|c|}{ Dor abdominal } \\
\hline sim & 6 & 37,5 & 13 & 52,0 & 1 & 12,5 & 18 & 54,5 \\
\hline não & 10 & 62,5 & 12 & 48,0 & 7 & 87,5 & 15 & 45,5 \\
\hline
\end{tabular}

AST/TGO: aspartato-aminotransferase. ALT/TGP: alanino-aminotransferase.

Tabela 2 - Avaliação das alterações hepáticas em 41 casos de dengue hemorrágico em Campo Grande/MS em 2002.

\begin{tabular}{|c|c|c|c|c|c|c|c|c|}
\hline \multirow{3}{*}{$\begin{array}{l}\text { Clínica } \\
\text { Exames }\end{array}$} & \multicolumn{2}{|c|}{ DH I } & \multicolumn{2}{|l|}{ DH II } & \multicolumn{2}{|l|}{ DH III } & \multicolumn{2}{|c|}{ DH IV } \\
\hline & $\mathrm{n}^{0}$ alter/ & & $\mathrm{n}^{0}$ alter/ & & $\mathrm{n}^{0}$ alter/ & & $\mathrm{n}^{0}$ alter/ & \\
\hline & $\mathrm{n}^{0}$ invest & alt $\%$ & $\mathrm{n}^{0}$ invest & alt $\%$ & $\mathrm{n}^{0}$ invest & alt $\%$ & $\mathrm{n}^{0}$ invest & alt $\%$ \\
\hline$\overline{\text { ALT }}$ & $2 / 3$ & 66,7 & $16 / 25$ & 64,0 & $6 / 12$ & 50,0 & $1 / 1$ & - \\
\hline AST & $2 / 3$ & 66,7 & $22 / 25$ & 88,0 & $8 / 12$ & 66,7 & $1 / 1$ & \\
\hline GGT & $0 / 1$ & - & $6 / 14$ & 42,8 & $2 / 5$ & 40,0 & - & - \\
\hline $\mathrm{FA}$ & $0 / 1$ & - & $1 / 14$ & 7,1 & $3 / 7$ & 42,8 & - & - \\
\hline PT & $0 / 2$ & - & $5 / 20$ & 25,0 & $4 / 10$ & 40,0 & $1 / 1$ & - \\
\hline ALB & $1 / 2$ & 50,0 & $9 / 20$ & 45,0 & $5 / 10$ & 50,0 & $1 / 1$ & - \\
\hline
\end{tabular}

AST: aspartato-aminotransferase; ALT: alanino-aminotransferase; GGT: gama-glutamiltransferase; FA: fosfatase alcalina; PT: proteínas totais; ALB: albumina; DH: dengue hemorrágico. 
ALT e 73,9\% (17/23) para a AST nas mulheres e 72,2\% (13/ 18) para ALT e $88,9 \%(16 / 18)$ para a AST nos homens, mas as diferenças encontradas não são estatisticamente significativas.

Nas sorologias para hepatites B e C encontramos um paciente com anti-HCV positivo confirmado por western blot, sendo que este paciente apresentava elevação nas enzimas hepáticas (ALT 71 U/l e AST 110 U/l) e 5 pacientes com HBSAg positivo (4 com confirmação por western blot), todos apresentavam alteração na AST que variou de 82 a 233 U/1 e 3 tinham elevação na ALT que variou do normal até 198 U/l.

\section{DISCUSSÃO}

As alterações hepáticas refletidas como hepatomegalia, aumento das enzimas hepáticas, hepatite fulminante e encefalopatia, já foram descritas tanto em casos de dengue clássico ${ }^{6}$, como em casos de dengue hemorrágico ${ }^{11}{ }^{12}$.

Grande parte dos estudos que demonstram as alterações hepáticas no dengue foram realizados em crianças: Nguyen cols ${ }^{12}$ estudaram crianças (não especificaram a idade); Lum cols ${ }^{11}$ estudaram crianças de 11 meses a 11 anos; Nimmannitya cols ${ }^{13}$, crianças de 2 meses a 13 anos; Rosen e cols ${ }^{16}$, crianças de 2 a 10 anos de idade.

No Brasil, Souza cols ${ }^{19}$ avaliaram adultos com dengue e encontraram $63,4 \%$ de alteração na AST e $45 \%$ na ALT, concluindo que a elevação das transaminases e a hepatite reativa são complicações comuns nos pacientes com infecção pelo vírus dengue.

A casuística apresentada constituída de adultos jovens vem, juntamente com outros estudos ${ }^{4}$, alertar para a ocorrência de complicações hepáticas nessa faixa etária, considerando também que é uma população que pode apresentar comorbidades como alcoolismo e outras hepatites virais.

$\mathrm{Na}$ investigação das manifestações clínicas que poderiam estar relacionadas a alterações da função hepática (náuseas e vômitos, dor abdominal e hepatomegalia) não encontramos diferenças estatisticamente significativas entre os pacientes que apresentavam enzimas alteradas (ALT e AST) e aqueles que tinham dosagem enzimática dentro da normalidade. Entre os sinais e sintomas investigados aquele que apresentou tendência a associar-se com aumento de AST foi a dor abdominal, que ocorreu em 12,5\% dos pacientes com AST normal e em 54,5\% dos pacientes com AST alterada $(\mathrm{p}=0,081086)$.

$\mathrm{Na}$ avaliação geral dos pacientes internados por dengue encontramos 90\% (37/41) de pacientes com queixa de náuseas e/ou vômitos. Zagne cols ${ }^{22}$, estudando 56 casos de $\mathrm{DH}$, encontraram $61 \%$ dos pacientes que apresentavam náuseas e 55\% vômitos. Cortiñas cols ${ }^{2}$, em 200 casos de dengue hemorrágico, observaram náuseas em 47 (23,5\%) pacientes e vômitos em 58 (29\%). Em nenhum desses dois trabalhos foi realizada a investigação de alterações hepáticas.

Com relação à dor abdominal, 46,3\% (19/41) dos nossos pacientes queixavam-se desse sintoma. Caballero cols ${ }^{1}$, estudando 77 crianças com dengue clássico durante a epidemia de 1997 em Cuba, encontraram 14 (18,1\%) que apresentavam dor abdominal. Na mesma epidemia, Díaz cols $^{4}$, em 37 pacientes adultos com hepatite reativa pelo DENV, observaram que $45,9 \%$ apresentavam dor em hipocôndrio direito e Cortiñas cols ${ }^{2}$, em 200 pacientes com DH, encontraram 152 (76\%) com dor abdominal.

0 sinal de hepatomegalia ao exame físico foi encontrado em $10 \%(3 / 29)$ dos nossos pacientes, sendo que em 1 deles as dosagens de ALT e AST eram normais. Estudos realizados em adultos cubanos detectaram hepatomegalia em frequiências variáveis, de $49,5 \%$ até $83,8 \%{ }^{24}$; em crianças, a freqüência foi bem menor, cerca de $5 \%^{1}$. Essas variações refletem as diferenças nas amostras em estudo, mas também as diferentes metodologias de trabalho.

Nos casos estudados observamos que 61\% (25/41) apresentavam alteração na ALT e 80,5\% (33/41) na AST. RigauPerez ${ }^{15}$, em 57 casos de DH, encontrou 70,8\% (34/48) de alteração na dosagem de ALT e 84\% (42/50) de alteração na AST. Nguyen cols $^{12}$, em 45 pacientes com DH, observaram 37,3\% de ALT alterada e $97,7 \%$ de AST acima do normal, com valores médios de 54,2U/1 (4,1-279U/1) para a ALT e de 128,4U/1 (24-279U/1) para a AST. A variação nos valores enzimáticos encontrados em nossos casos foi 14-547U/1 para a ALT com média de 89,7U/1 e de 11-298U/1 para a AST com média de 104,5U/.

0 tratamento realizado durante a internação foi a vigilância das alterações hemodinâmicas com hidratação precoce e medicações sintomáticas de suporte. Todos os pacientes apresentaram regressão dos sintomas e retornaram as suas atividades normais.

No Brasil, os relatos de estudos clínicos do dengue são escassos e há poucas referências às alterações hepáticas da doença entre nós.

A alta porcentagem de casos com alterações nas enzimas hepáticas encontradas neste estudo traz à luz a importância da avaliação hepática nos pacientes com dengue e $\mathrm{DH}$. No entanto, muito ainda temos a compreender das inter-relações entre os DENV e o fígado, como, por exemplo, sua ação em órgãos já comprometidos por outras hepatites virais crônicas como as hepatites $\mathrm{B}$ e C, ou em fígados comprometidos pelo álcool ou medicamentos, ou ainda em pacientes com hepatites auto-imunes.

Há que se compreender melhor também a própria ação dos DENV na célula hepática e sua evolução com a doença em pacientes previamente hígidos e naqueles portadores de doença crônica não relacionada ao fígado, razão pela qual outros estudos serão necessários para elucidar estas questões.

\section{AGRADECIMENTOS}

Os autores agradecem à Prof ${ }^{a}$ Ana Rita Coimbra Motta de Castro, da UFMS, pela realização das sorologias para hepatites $\mathrm{B}$ e C, e à Dra. Marize Pereira Miagostovich, do Instituto Oswaldo Cruz/FIOCRUZ, pelo apoio técnico durante a realização da pesquisa. 


\section{REFERÊNCIAS BIBLIOGRÁFICAS}

1. Caballero MEV, Portuondo TMA, Serrano HP. Características clínicas de la fiebre de dengue en niños durante el brote epidémico en Santiago de Cuba. Revista Cubana de Medicina Tropical 53: 20-23, 2001.

2. Cortiñas MG, González DV, Cordero JC, Oliveiras MLL. Dengue hemorrágico. Estudo clínico de 200 pacientes. Revista Cubana de Medicina 38: 13-18, 1999.

3. Cunha RV, Marques Filho VS, Aguiar JIA, Paniago AMM, Lindenberg ASC, Hans Filho G, Miagostovich MP, Nogueira RMR, Schatzmayr HG. Dengue Hemorrágico em Campo Grande, MS: Relato do primeiro caso diagnosticado. In: Resumos do XXXII Congresso da Sociedade Brasileira de Medicina Tropical, Goiânia, 1996. Revista da Sociedade Brasileira de Medicina Tropical 29 (supl 1): 57, 1996.

4. Díaz SV, Martínez MP, Saent-Félix FG. Hepatitis reactiva por virus del dengue hemorrágico. Revista Cubana de Medicina Tropical 53: 28-31, 2001.

5. Gadelha DP, Toda AT. Biologia e comportamento do Aedes aegypti. Revista Brasileira de Malariologia e Doenças Tropicais 37: 29-36, 1985.

6. George R, Liam CK, Chua CT, Lam SK, Pang T, Geethan R, Fools. Unusual clinical manifestations of dengue virus infection. The Southeast Asian Journal Tropical Medicine and Public Health 19: 585-590, 1988

7. Gubler DJ. Epidemic dengue/dengue hemorrhagic fever as a public health, social and economic problem in the 21st century. Trends in Microbiology 10: 100-103, 2002.

8. Guzmán MG, Kourí G. Dengue: an update. The Lancet Infectious Diseases 2: $33-42,2002$

9. Halstead SB. Dengue. Current Opinion in Infectious Diseases 15: 471-76, 2002.

10. Hammon WM, Sather GE. Virological findings in the 1960 hemorrhagic fever epidemic (dengue) in Thailand. American Journal of Tropical Medicine and Hygiene 13: 629-641, 1960.

11. Lum LCS, Lam SK, George R, Devi S. Fulminant Hepatitis in Dengue Infection. The Southeast Asian Journal Tropical Medicine and Public Health 24: 467-471, 1993.
12. Nguyen TL, Nguyen TH, Tieu NT. The impact of dengue haemorrhagic fever on liver function. Research in Virology 148: 273-277, 1997.

13. Nimmannitya S, Thisyakorn U, Hemsrichart Y. Dengue Haemorrhagic Fever with unusual manifestations. The Southeast Asian Journal of Tropical Medicine and Public Health 18: 398-405, 1987.

14. Nogueira RMR, Filippis AM, Coelho JM, Sequeira PC, Schatzmayr HG, Paiva FG, Ramos AM, Miagostovich MP. Dengue vírus infection of the central nervous system (CNS): A case report from Brazil. The Southeast Asian Journal of Tropical Medicine and Public Health 33: 68-71, 2002

15. Rigau-Perez JG. Manifestações clínicas del dengue hemorágico em Puerto Rico, 1990-1991. Revista Panamericana de Salud Pública 1: 435-443, 1997.

16. Rosen L, Khin MM, Tin U. Recovery of virus from the liver of children with fatal dengue: reflections on the pathogenesis of the disease and its possible analogy with that of yellow fever. Research Virology 140: 351-360, 1989.

17. Sabin AB. Research on dengue during World War II. American Journal of Tropical Medicine and Hygiene 1: 30-50, 1952

18. Schlesinger RW, Frankel JW. Adaptation of the New Guinea B strains of dengue virus to suckling mice and to adults Swiss mice. Journal Immunology 77: 352-363, 1956.

19. Souza LJ, Alves JG, Nogueira RM, Gicoyate Neto C, Bastos DA, Siqueira EW, Souto Filho JT, Cezario TA, Soares CE, Carneiro RC. Aminotransferase Changes and Acute Hepatitis in Patients With Dengue Fever: Analysis of 1,585 Cases. The Brazilian Journal of Infection Diseases 8: 156-163, 2004.

20. Westaway EG, Brinton MA, Gaidamovich SY, Horzinek MC, Igarashi A Kaariainen L, Lvov DK, Porterfield JS, Russell PK, Trent DW. Flaviviridae. Intervirology 24: 183-192, 1985.

21. World Health Organization. Dengue haemorrhagic fever: diagnosis, treatment and control. 2a edition. Geneva, p.1-87, 1997.

22. Zagne SMO, Alves VG, Nogueira RM, Miagostovich MP, Lampe E, Tavares W. Dengue haemorrhagic fever in the state of Rio de Janeiro, Brazil: a study of 56 confirmed cases. Transactions of the Royal Society of Tropical Medicine and Hygiene 88: 677-679, 1994 